\title{
Chronos heute
}

\section{Zeitreisen in Zeiten ohne Zeit}

\author{
Renate Sgier
}

Online publiziert: 21. August 2015

(C) Springer-Verlag Berlin Heidelberg 2015

In der mittelalterlichen Zähringerstadt Bern fand vom 25. bis zum 28.09.2014 die 6. Deutschsprachige Internationale Psychoanalytische Tagung (DIPSAT), die die Mitteleuropäische Tagung abgelöst hat, statt. Nach der Tradition lag der Schwerpunkt neben der Diskussion eines spezifischen psychoanalytischen Themas auch in Bern auf der klinischen Arbeit in Gestalt von Fallsupervisionen, die den Austausch und den Dialog zwischen unterschiedlichen theoretischen und technischen Ansätzen zu einer Bereicherung werden lässt. Ebenso spezifisch für diese Tagung ist die Tatsache, dass Kandidaten bzw. Analytiker in Ausbildung aus den verschiedenen deutschsprachigen Gesellschaften sich in Koreferaten zu den Hauptvorträgen äußern.

Das Tagungsthema, kreisend um Chronos, um Zeitreisen in Zeiten ohne Zeit, macht auf den vordergründigen Widerspruch aufmerksam, zwischen der oft mit langen Umwegen verbundenen analytischen Kur und der von Beschleunigung, Effizienz und der Illusion einer perfekten Zukunft und von ewigem Jungsein geprägten heutigen Gesellschaft. Diese entpuppen sich bei näherem Hinsehen jedoch meist als Phänomene, denen wir in der Kur begegnen, und muten wie „Zeitreisen“ an, wie Einbrüche von ,zeitlosen“ Wünschen und Fantasien, die der Realität, der Gegenwart, dem bewussten Wahrnehmen der ablaufenden Zeit ein „Schnippchen“ zu schlagen suchen.

Wiederholungen, Aktualisierungen in der Kur von unbewussten, nach Freud ,zeitlosen" Wünschen und Fantasien, die in der Übertragung, im Agieren zum Zuge kommen, bringen etwas in die Gegenwart, was nur vordergründig gegenwärtig ist, denn, wenn es Gegenwart wäre, würde es zur Vergangenheit. Es wiederholt sich etwas immer wieder, gerade weil es nicht vorgestellt, nicht erkannt, nicht betrauert und dadurch zur Vergangenheit werden kann. Das Wiederholte ist weder Vergangenheit

R. Sgier $(\bowtie)$

Schweizerische Gesellschaft für Psychoanalyse,

Oberer Schmittenweg 33,

4914 Roggwil, Schweiz

E-Mail: renasgier@gmail.com 
noch Gegenwart; es gehört einer ewigen, einer ,zeitlosen Gegenwart“ an, gehört den „unbewussten, zeitlosen Prozessen, welche nicht zeitlich geordnet sind und die durch die Zeit nicht verändert werden, ja welche absolut keine Beziehung zur Zeit haben“" (Freud 1915, S. 286). Zeitlosigkeit, oder „eine andere Zeit“, wie es Jean-Bertrand Pontalis (zit. nach Green 2001) bezeichnet, als eine Zeit, die nicht vergeht? Green (2001) setzt die Zeitlosigkeit des Unbewussten mit der Zeitlosigkeit des Eros gleich, mit der Nachhaltigkeit der Spuren, die in der frühen Kindheit angelegt wurden in der Psyche und immer aktiv bleiben. Neue assoziative Verbindungen zu schaffen und den ,zeitlosen Phänomenen“ zu ermöglichen, Gegenwart und dann Vergangenheit zu werden, ist eine Aufgabe der Psychoanalyse. Faimberg (2005) beschreibt in ihrem Buch: Teleskopieren der Generationen, wie durch Entidentifizierungen der für die Psychoanalyse wesentliche Prozess der Historisierung eingeleitet wird.

Viele interessante Überlegungen zu Zeit und Zeitreisen in der Kur wurden während dieser Tagung entwickelt und diskutiert, und die Vorträge und Koreferate sind, falls es die Wahrung der Diskretion erlaubt hat, in diesem Heft publiziert.

\section{Literatur}

Faimberg H (2005) Telescoping of generations: listening to the narcissistic links between generations. Routledge, London

Freud S (1915) Das Unbewusste. GW, Bd 10

Green A (2001) Le temps éclaté. In: RFP, Bd 65

Renate Sgier, von 2012 bis 2015 Präsidentin der Schweizerischen Gesellschaft für Psychoanalyse (SGPsa). 